\title{
Delay of the Blink Reflex in Patients Receiving Platinum-Analogue Chemotherapy
}

\author{
Kang Young Park, $\mathrm{MD}^{1}$, Young Sook Park, $\mathrm{MD}^{1}$, Yun Hee Park, $\mathrm{MD}^{1}$, Hyun Jung Chang, $\mathrm{MD}^{1}$, \\ Eun Sol Cho, $\mathrm{MD}^{1}$, Seok-Hyun Kim, $\mathrm{MD}, \mathrm{PhD}^{2}$, Woo Jin Kim, $\mathrm{MD}^{3}$
}

\begin{abstract}
${ }^{1}$ Department of Physical Medicine and Rehabilitation, Samsung Changwon Hospital, Sungkyunkwan University School of Medicine, Changwon; ${ }^{2}$ Division of Hematology and Medical Oncology, Department of Internal Medicine, Samsung Changwon Hospital, Sungkyunkwan University School of Medicine, Changwon; ${ }^{3}$ Department of Physical Medicine and Rehabilitation, Haeundae Paik Hospital, Inje University School of Medicine, Busan, Korea
\end{abstract}

Objective To investigate the presence of cranial neuropathy in patients with platinum-analogue chemotherapy using electrodiagnostic evaluations.

Methods Thirty-nine patients whose chemotherapy was completed within a month and 40 control subjects were enrolled in the study. Electrodiagnostic evaluation was performed using sensory and motor nerve conduction studies and blink reflex studies, in addition to the two-point discrimination test.

Results The chemotherapy group had significantly longer latencies of bilateral R1 responses (left $\mathrm{p}<0.001$; right $\mathrm{p}<0.001)$ and greater distance in two-point discrimination $(\mathrm{p}<0.001)$ compared to the control group. In the subgroup with peripheral polyneuropathy, the left $\mathrm{R} 1 \mathrm{p}=0.01$ ), both $\mathrm{R} 2 \mathrm{i}$ (left $\mathrm{p}=0.02$; right $\mathrm{p}=0.03$ ) and the left $\mathrm{R} 2 \mathrm{c}$ ( $\mathrm{p}=0.02)$ were prolonged relative to those without the polyneuropathy, and both $\mathrm{R} 1$ (left $\mathrm{p}<0.001$; right $\mathrm{p}<0.001$ ), R2i (left $\mathrm{p}=0.01$; right $\mathrm{p}=0.03)$, and the left $\mathrm{R} 2 \mathrm{c}(\mathrm{p}=0.01)$ were prolonged relative to the controls. On the other hand, the subgroup without the polyneuropathy showed only prolongation of both R1 (left $\mathrm{p}=0.006$; right $\mathrm{p}<0.001$ ) relative to the controls.

Conclusion In the present study, comparison of blink reflex and two-point discrimination showed the likelihood of subclinical cranial neuropathy following platinum-analogue chemotherapy. Cranial neuropathy caused by platinum agents was more profound in patients with peripheral polyneuropathy and may be dependent on the cumulative dose of the drug. The blink reflex may be of value in detecting subclinical cranial neuropathy in patients undergoing platinum-analogue chemotherapy.

Keywords Platinum compounds, Chemotherapy, Blink, Cranial nerve

Received May 29, 2015; Accepted July 27, 2015

Corresponding author: Young Sook Park

Department of Physical Medicine and Rehabilitation, Samsung Changwon Hospital, Sungkyunkwan University School of Medicine, 158 Paryong-ro, Masanhoewon-gu, Changwon 51353, Korea. Tel: +82-55-290-6390, Fax: +82-55-290-6588, E-mail: jijibaeheiwon@hanmail.net

() This is an open-access article distributed under the terms of the Creative Commons Attribution Non-Commercial License (http://creativecommons.org/ licenses/by-nc/4.0) which permits unrestricted noncommercial use, distribution, and reproduction in any medium, provided the original work is properly cited. Copyright $\odot 2016$ by Korean Academy of Rehabilitation Medicine 


\section{INTRODUCTION}

Platinum-analogues, a type of DNA alkylating agent, are important chemotherapeutic drugs which form the basis for treatment for many solid tumor types, including lung, colorectal, gastric, esophageal, head and neck, cervical, bladder, ovarian, and testicular cancer [1]. However, these agents are known to cause peripheral polyneuropathy as do the taxanes, epothilones, vinca alkaloids, and bortezomib [2]. Chronic neuro-sensory toxicities caused by these drugs are a major known side effect presenting clinically as distal dysesthesia, pain in glove and stocking forms, decrease in the deep tendon reflex (DTR), and reduced peripheral sensation, ototoxicity, and hearing loss [3-5].

While there have been frequent reports of peripheral polyneuropathies or central nervous system involvement resulting from the use of platinum-analogues, reports of cranial neuropathies are rare. Verschraegen et al. [6] reported visual loss, which occurred after treatment with cisplatin, but this was a symptom reflecting involvement of the cerebral visual cortex and not from peripheral (optic nerve) cranial neuropathy. Some literature have reported that platinum-analogues may induce cranial neuropathy, but most such publications on cranial nerve defects due to platinum therapy are in the form of case reports [7]. Accordingly, we performed a control group study using the blink reflex on patients who received platinum-analogue chemotherapy and intended to examine whether a cranial neuropathy could be caused by these drugs.

\section{MATERIALS AND METHODS}

\section{Subjects}

\section{Chemotherapy group}

The adult patients aged 18 or older who completed platinum-analogue chemotherapy in the division of hematology-oncology, department of internal medicine who agreed to participate in this study were consecutively recruited and enrolled from April 2013 to January 2014. All the evaluations were finished within one month following the last cycle of the chemotherapy. In order to minimize the effects of the complex chemotherapy regimen, patients who used other chemotherapeutic agents, not including 5-fluorouracil (5-FU) or etoposide, were excluded from this study. In addition, those who had a history of head and/or neck trauma, systemic illness which can cause peripheral polyneuropathy (e.g., diabetes), and/or central or peripheral nervous system (CNS or PNS) disorders (e.g., stroke) were excluded.

\section{Control group}

Among adult patients who received electrodiagnostic studies for the evaluation of a radiculopathy in the Department of Physical Medicine and Rehabilitation at Samsung Changwon Hospital, Sungkyunkwan University School of Medicine, those who agreed to participate in the study as a control group were consecutively recruited during the same time period as for the chemotherapy group. Those who had abnormal findings in the nerve conduction study (NCS), had a history of head and neck trauma, systemic illness that can cause peripheral polyneuropathy, or a CNS or PNS disorders were excluded.

\section{Methods}

\section{Electrodiagnostic studies}

Electrodiagnostic studies were performed using the Viking electromyography system (Nicolet, Middleton, WI, USA) and surface electrodes. The infraorbital blink reflex study and NCS on the peripheral nerves of both the upper and lower limbs were performed bilaterally in all subjects.

For the blink reflex study, patients were placed in a supine position with eyes closed comfortably with the active electrode attached to the orbicularis oculi muscle, the reference electrodes to the bilateral temples, the lateral electrode to the eye, and the ground electrode to the chin. The stimulation intensity was set at 20-25 mA with a duration of $0.1 \mathrm{~ms}$ at a frequency of $0.5 \mathrm{~Hz}$ to stimulate the infraorbital foramen. The frequency filtering range, sweep speed, and sensitivity were set at $20-200 \mathrm{~Hz}, 10$ $\mathrm{ms} /$ division, and $200 \mu \mathrm{V} /$ division, respectively. The latencies of the early (R1), ipsilateral (R2i), and contralateral (R2c) late responses were bilaterally measured.

NCS was composed of the following: motor NCS and Fwave study on the bilateral median, ulnar, peroneal, and tibial nerves; sensory NCS on the bilateral median, ulnar, superficial peroneal, and sural nerves; H-reflex test on bilateral tibial nerves. Sensory NCS was performed using antidromic conduction. When an abnormal finding was detected in two separate nerves or more, the subject was 
diagnosed with peripheral polyneuropathy [8].

\section{Two-point discrimination test}

Each of the bilateral cheeks of the patients with eyes were closed comfortably was stimulated twice with the A9292 two-point aesthesiometer (Lafayette Instrument Co., Lafayette, IN, USA). The minimum distance at which the patient perceived the two needles was measured in units of millimeters, and the average of the distances measured from both sides was derived.

\section{Data collection}

The patients' age and sex were collected through the medical record. In the chemotherapy group, the primary site of cancer, treatment regimen, and total duration of chemotherapy, cumulative dose of platinum agents, and period from the completion of chemotherapy to the electrodiagnostic study were collected additionally. Bilateral $\mathrm{R} 1, \mathrm{R} 2 \mathrm{i}$, and R2c latencies and presence of peripheral polyneuropathy were obtained from the results of electrodiagnostic studies. In addition, the results of the twopoint discrimination test were collected.

All of these protocols were approved by the Institution Review Board of our hospital.

\section{Statistical analysis}

The present study compared the results of the blink reflex study and two-point discrimination test between the chemotherapy and control groups as the first analysis. For the second analysis, we divided the chemotherapy group into subgroups according to the types of platinum agents and the presence of peripheral polyneuropathy and subsequently compared the results among each subgroups and the control group.

The Kolmogorov-Smirnov test was performed for age, total duration of chemotherapy, cumulative dose of platinum agents, period between the completion of chemotherapy and the electrodiagnostic study, latencies of bilateral R1, R2i, and R2c responses and mean distance of two-point discrimination. According to the test of normality, age, cumulative dose of platinum agents and latencies of bilateral R1, R2i, and R2c responses were reported as mean \pm standard deviation, and betweengroup comparison was carried out with the independent t-test or analysis of variance (ANOVA). Total duration of chemotherapy, the period from the completion of chemotherapy to the electrodiagnostic study, and mean distance of two-point discrimination were reported using median and interquartile range (IQR), and were compared using the Mann Whitney U-test or Kruskal-Wallis test. Categorical variables, such as sex, the primary site of cancer, and chemotherapy, were reported as frequency and proportion and descriptively analyzed.

The SPSS program for Windows ver. 18.0 (SPSS Inc., Chicago, IL, USA) was used for all the statistical analyses and p-values less than 0.05 were considered statistically significant.

\section{RESULTS}

A total of 39 patients (mean age $61.8 \pm 8.9$ years, 28 males) participated in the present study in the chemotherapy group. The numbers of those whose primary site of cancer was colon and rectum, lung, esophagus, stomach, and larynx were $15,10,7,6$, and 1, respectively. The numbers of those who used 5-fluorouracil (5-FU) and etoposide together with platinum agents were 30 and 9, respectively. The control group consisted of 40

Table 1. General characteristics of the chemotherapy and the control groups

\begin{tabular}{lcc}
\hline & $\begin{array}{c}\text { Chemotherapy } \\
(\mathbf{n}=\mathbf{3 9})\end{array}$ & $\begin{array}{c}\text { Control } \\
(\mathbf{n}=\mathbf{4 0})\end{array}$ \\
\hline Age (yr) & $61.8 \pm 8.9$ & $58.6 \pm 9.6$ \\
Male & $28(72)$ & $27(68)$ \\
Cancer diagnosis & & \\
$\quad$ Colon & $15(38)$ & - \\
\hline Lung & $10(26)$ & - \\
\hline Esophagus & $7(18)$ & - \\
\hline Stomach & $6(15)$ & - \\
\hline Larynx & $1(3)$ & - \\
CT Regimen & & \\
$\quad$ with 5-FU & $30(77)$ & - \\
\hline$\quad$ with etoposide & $9(23)$ & - \\
Duration of CT (day) & $63(38-183)$ & - \\
\hline Dose of drugs (mg/m $\left.{ }^{2}\right)$ & $658.2 \pm 522.0$ & - \\
Duration to EDX study (day) & $13(1-24)$ & - \\
\hline
\end{tabular}

Values are presented as mean \pm standard deviation or number (\%) or median (interquartile range).

CT, chemotherapy; 5-FU, 5-fluorouracil; EDX, electrodiagnostic. 
patients (mean age 58.6 \pm 9.6 years, 27 males) (Table 1). In comparing the control and chemotherapy groups, bilateral R1 latencies were significantly prolonged (the left side, $11.9 \pm 1.2 \mathrm{~ms}$ vs. $10.5 \pm 0.9 \mathrm{~ms}, \mathrm{p}<0.001$; the right side, $11.8 \pm 1.1 \mathrm{~ms}$ vs. $10.4 \pm 0.9 \mathrm{~ms}, \mathrm{p}<0.001$ ) and the mean distance of two-point discrimination was significantly longer (median [IQR] distance, $15.5 \mathrm{~mm}$ [12.5-18.5 mm] vs. $8 \mathrm{~mm}[6-9.375 \mathrm{~mm}] ; \mathrm{p}<0.001)$ in the chemotherapy group (Table 2).

The chemotherapy group was divided into two subgroups according to the type of platinum agent: $18 \mathrm{pa}-$ tients with cisplatin and 21 with oxaliplatin. Between the two subgroups, the cumulative dose of the agents signifi- cantly differed $\left(432.7 \pm 416.7 \mathrm{mg} / \mathrm{m}^{2}\right.$ vs. $851.4 \pm 534.0 \mathrm{mg} /$ $\mathrm{m}^{2} ; \mathrm{p}=0.01$ ), but there was no significant difference when the results of the blink reflex study and two-point discrimination test were compared. In both subgroups, bilateral R1 latencies were significantly prolonged (cisplatin vs. control: the left side, $11.9 \pm 1.0$ vs. $10.5 \pm 0.9$, $\mathrm{p}<0.001$; the right side, $11.9 \pm 0.9$ vs. $10.4 \pm 0.9, \mathrm{p}<0.001$; oxaliplatin vs. control: the left side, $11.9 \pm 1.4$ vs. $10.5 \pm 0.9, \mathrm{p}<0.001$; the right side, $11.6 \pm 1.3$ vs. $10.4 \pm 0.9, \mathrm{p}<0.001)$ and the mean distance of two-point discrimination was longer (cisplatin vs. control: median [IQR] distance, $14.75 \mathrm{~mm}$ [10.375-18.875 mm] vs. $8 \mathrm{~mm}[6-9.375 \mathrm{~mm}], \mathrm{p}<0.001$; oxaliplatin vs. control: $16 \mathrm{~mm}$ [12.75-18.5 mm] vs. $8 \mathrm{~mm}$

Table 2. Comparison between the chemotherapy and the control groups

\begin{tabular}{|c|c|c|c|}
\hline & Chemotherapy & Control & p-value \\
\hline \multicolumn{4}{|c|}{ Results of EDX study (ms) } \\
\hline Left R1 & $11.9 \pm 1.2$ & $10.5 \pm 0.9$ & $0.000^{\mathrm{a})}$ \\
\hline Left R2i & $29.1 \pm 5.8$ & $27.5 \pm 3.5$ & 0.154 \\
\hline Left R2c & $31.8 \pm 6.0$ & $29.9 \pm 3.7$ & 0.109 \\
\hline Right R1 & $11.8 \pm 1.1$ & $10.4 \pm 0.9$ & $0.000^{\mathrm{a})}$ \\
\hline Right R2i & $29.0 \pm 5.7$ & $27.6 \pm 3.6$ & 0.202 \\
\hline Right R2c & $31.3 \pm 6.3$ & $29.8 \pm 4.0$ & 0.225 \\
\hline TPD test $(\mathrm{mm})$ & $15.5(12.5-18.5)$ & $8(6-9.375)$ & $0.000^{\mathrm{b})}$ \\
\hline
\end{tabular}

Values are presented as mean \pm standard deviation or median (interquartile range).

EDX, electrodiagnostic; R2i, ipsilateral R2; R2c, contralateral R2; TPD, two-point discrimination.

${ }^{a)} \mathrm{p}<0.05$ by independent $\mathrm{t}$-test, ${ }^{\text {b) }} \mathrm{p}<0.05$ by Mann-Whitney U-test.

Table 3. Comparison among the control group and the patients treated with cisplatin and oxaliplatin

\begin{tabular}{|lccc}
\hline & Control $(\mathbf{n}=\mathbf{4 0})$ & Cisplatin $(\mathbf{n}=\mathbf{1 8})$ & Oxaliplatin $(\mathbf{n}=\mathbf{2 1})$ \\
\hline Age $(\mathrm{yr})$ & $58.6 \pm 9.6$ & $59.3 \pm 8.2$ & $63.9 \pm 9.1$ \\
\hline Duration of CT $($ day) & - & $61(29.5-189.25)$ & $63(41-179)$ \\
\hline Dose of drugs $\left(\mathrm{mg} / \mathrm{m}^{2}\right)$ & - & $432.7 \pm 416.7$ & $\left.851.4 \pm 534.0^{\mathrm{a}}\right)$ \\
\hline Duration to EDX study (day) & - & $7.5(1-26.25)$ & $17(2.5-23)$ \\
\hline Results of EDX study $(\mathrm{ms})$ & & & \\
\hline Left R1 & $10.5 \pm 0.9$ & $11.9 \pm 1.0^{\mathrm{b})}$ & $11.9 \pm 1.4^{\mathrm{b})}$ \\
\hline Left R2i & $27.5 \pm 3.5$ & $28.9 \pm 5.7$ & $29.2 \pm 6.0$ \\
\hline Left R2c & $29.9 \pm 3.7$ & $31.5 \pm 5.7$ & $32.0 \pm 6.4$ \\
\hline Right R1 & $10.4 \pm 0.9$ & $11.9 \pm 0.9^{\mathrm{b})}$ & $11.6 \pm 1.3^{\mathrm{b})}$ \\
\hline Right R2i & $27.6 \pm 3.6$ & $29.0 \pm 5.7$ & $29.0 \pm 5.9$ \\
\hline Right R2c & $29.8 \pm 4.0$ & $31.3 \pm 5.5$ & $31.2 \pm 7.0$ \\
\hline TPD test $(\mathrm{mm})$ & $8(6-9.375)$ & $14.75(10.375-18,875)^{\mathrm{b})}$ & $16(12.75-18.5)^{\mathrm{b})}$ \\
\hline
\end{tabular}

Values are presented as mean \pm standard deviation or median (interquartile range).

CT, chemotherapy; EDX, electrodiagnostic; R2i, ipsilateral R2; R2c, contralateral R2; TPD, two-point discrimination.

${ }^{a)} \mathrm{p}<0.05$ compared to the cisplatin group by independent $\mathrm{t}$-test, ${ }^{\mathrm{b})} \mathrm{p}<0.05$ compared to the control group by analysis of variance or Kruskal-Wallis test. 
[6-9.375 mm], $\mathrm{p}<0.001$ ) than in the control group (Table 3). Also, the chemotherapy group was subdivided into two smaller subgroups according to the presence of peripheral polyneuropathy; 20 patients were included in the group with polyneuropathy and 19 in the group without. Those with polyneuropathy were significantly older (64.6 \pm 9.4 years vs. $58.8 \pm 7.5$ years; $p=0.04)$, underwent a longer duration of chemotherapy (median [IQR] duration, 171 days [47.75-199 days] vs. 57 days [30-65 days]; $\mathrm{p}=0.008$ ), higher cumulative dose of platinum agents (887.4 $\pm 546.9 \mathrm{mg} / \mathrm{m}^{2}$ vs. $\left.417.0 \pm 373.7 \mathrm{mg} / \mathrm{m}^{2} ; \mathrm{p}=0.003\right)$, had a longer period from the completion of chemotherapy to the electrodiagnostic study (median [IQR] period, 22.5 days [4-28.5 days] vs. 4 days [1-21 days]; $\mathrm{p}=0.008$ ), longer latencies of the left R1 $(12.4 \pm 1.2 \mathrm{~ms}$ vs. $11.4 \pm 1.0$ ms; $\mathrm{p}=0.01)$, R2i ( $31.1 \pm 6.2 \mathrm{~ms}$ vs. $26.9 \pm 4.5 \mathrm{~ms} ; \mathrm{p}=0.02)$, R2c (33.8 $\pm 6.5 \mathrm{~ms}$ vs. $29.6 \pm 4.6 \mathrm{~ms} ; \mathrm{p}=0.02)$, and the right R2i (30.9 $\pm 6.2 \mathrm{~ms}$ vs. $27.0 \pm 4.5 \mathrm{~ms} ; \mathrm{p}=0.03)$ responses, and greater distance of two-point discrimination (median [IQR] distance, $17.5 \mathrm{~mm}$ [15.625-19.75 mm] vs. $13 \mathrm{~mm}$ [10.5-15.5 mm]; $p=0.008)$. In the subgroup without the polyneuropathy, bilateral R1 latencies were significantly prolonged (the left side, $11.4 \pm 1.0$ vs. $10.5 \pm 0.9, \mathrm{p}=0.006$; the right side, $11.4 \pm 0.9$ vs. $10.4 \pm 0.9, \mathrm{p}<0.001$ ), and the mean distance of two-point discrimination was longer (median [IQR] distance, $13 \mathrm{~mm}$ [10.5-15.5 mm] vs. $8 \mathrm{~mm}$
[6-9.375 $\mathrm{mm}] ; \mathrm{p}<0.001)$ than in the control group. In the subgroup with the polyneuropathy, bilateral Rl (the left side, $12.4 \pm 1.2$ vs. $10.5 \pm 0.9, \mathrm{p}<0.001$; the right side, $12.1 \pm 1.2$ vs. $10.4 \pm 0.9, \mathrm{p}<0.001$ ), R2i (the left side, $31.1 \pm 6.2$ vs. $27.5 \pm 3.5$, $\mathrm{p}=0.01$; the right side, $30.9 \pm 6.2$ vs. $27.6 \pm 3.6$, $\mathrm{p}=0.03)$, the left R2c (33.8 \pm 6.5 vs. $29.9 \pm 3.7, \mathrm{p}=0.01)$ latencies, and mean distance of two-point discrimination (median [IQR] distance, $17.5 \mathrm{~mm}$ [15.625-19.75 mm] vs. $8 \mathrm{~mm}[6-9.375 \mathrm{~mm}] ; \mathrm{p}<0.001)$ showed significant differences compared to the control group (Table 4).

\section{DISCUSSION}

Platinum-derivatives are important chemotherapeutic agents that form the mainstay for treatment of lung, colorectal, gastric, head and neck, cervical, esophageal, bladder, ovarian, and testicular cancer in combination with radiation therapy and/or other chemotherapeutic agents [1]. Since 1978, when cisplatin, the first-generation platinum agent, was approved for the treatment of ovarian and testicular cancer [9], development of other platinum analogues has developed into forms associated with less toxicity and greater treatment efficacy. Carboplatin is a second-line platinum chemotherapeutic agent used for the treatment of lung cancer in combination with gemcitabine or paclitaxel [10], and the third-generation ox-

Table 4. Comparison among the control group and the patients with and without PPN

\begin{tabular}{|lccc}
\hline & Control $(\mathbf{n}=\mathbf{4 0})$ & Without PPN $(\mathbf{n}=\mathbf{1 9})$ & With PPN $(\mathbf{n}=\mathbf{2 0})$ \\
\hline Age (yr) & $58.6 \pm 9.6$ & $58.8 \pm 7.5$ & $64.6 \pm 9.4^{\mathrm{b})}$ \\
\hline Duration of CT (day) & - & $57(30-65)$ & $171(47.75-199)^{\mathrm{a})}$ \\
\hline Dose of drugs $\left(\mathrm{mg} / \mathrm{m}^{2}\right)$ & - & $417.0 \pm 373.7$ & $887.4 \pm 546.9^{\mathrm{a})}$ \\
\hline Duration to EDX study (day) & - & $4(1-21)$ & $22.5(4-28.5)^{\mathrm{a})}$ \\
\hline Results of EDX study (ms) & & & \\
\hline Left R1 & $10.5 \pm 0.9$ & $11.4 \pm 1.0^{\mathrm{b})}$ & $12.4 \pm 1.2^{\mathrm{b}, \mathrm{c})}$ \\
\hline Left R2i & $27.5 \pm 3.5$ & $26.9 \pm 4.5$ & $31.1 \pm 6.2^{\mathrm{b}, \mathrm{c})}$ \\
\hline Left R2c & $29.9 \pm 3.7$ & $29.6 \pm 4.6$ & $33.8 \pm 6.5^{\mathrm{b}, \mathrm{c})}$ \\
\hline Right R1 & $10.4 \pm 0.9$ & $11.4 \pm 0.9^{\mathrm{b})}$ & $12.1 \pm 1.2^{\mathrm{b})}$ \\
\hline Right R2i & $27.6 \pm 3.6$ & $27.0 \pm 4.5$ & $30.9 \pm 6.2^{\mathrm{b}, \mathrm{c})}$ \\
\hline Right R2c & $29.8 \pm 4.0$ & $29.5 \pm 4.5$ & $32.9 \pm 7.3$ \\
\hline TPD test (mm) & $8(6-9.375)$ & $13(10.5-15.5)^{\mathrm{b})}$ & $17.5(15.625-19.75)^{\mathrm{b}, \mathrm{c})}$ \\
\hline
\end{tabular}

Values are presented as mean \pm standard deviation or median (interquartile range).

PPN, peripheral polyneuropathy; CT, chemotherapy; EDX, electrodiagnostic; R2i, ipsilateral R2; R2c, contralateral R2; TPD, two-point discrimination.

${ }^{a)} \mathrm{p}<0.05$ compared to the patients without PPN by independent $\mathrm{t}$-test or Mann-Whitney U-test, ${ }^{\text {b) }} \mathrm{p}<0.05$ compared to the control group by analysis of variance or Kruskal-Wallis test, ${ }^{c} \mathrm{p}<0.05$ compared to the patients without PPN by analysis of variance or Kruskal-Wallis test. 
aliplatin is utilized for treating colorectal cancer together with 5-FU and leucovorin [11]. Neurotoxicity of platinum agents and the consequent peripheral polyneuropathy remains a major non-hematological dose-limiting side effect. Although research for the prevention or minimization of polyneuropathy (e.g., glutathione and vitamin E) is continuing, there has yet been no development of effective treatment or prevention [12].

Cranial neuropathy resulting from anti-cancer treatment has been reported to be associated with chemotherapeutic agents such as vincristine, cyclosporine, and cytarabine [7]. Toker et al. [13] reported diplopia caused by abducens nerve paralysis following chemotherapy with vincristine for three weeks in a 28-year-old nonHodgkin's lymphoma patient. Vogel and Horoupian [14] reported dysarthria and ophthalmoplegia which occurred after high-dose cytarabine treatment in a 43-yearold patient with acute promyelocytic leukemia and was found to have involvement of the brainstem and spinal motor neuron. Verschraegen et al. [6] reported a case of blindness, which occurred after cisplatin treatment, but this was determined to be due to involvement of the cerebral visual cortex rather than by a cranial neuropathy. Research on peripheral polyneuropathy or CNS side effects resulting from chemotherapy have been frequently reported. However, the literature on platinum chemotherapy-associated cranial neuropathy are limited to few, isolated case reports. Accordingly, the authors planned this control group study in order to evaluate whether cranial neuropathies could be caused by platinum agents. In the present study, the chemotherapy group showed delay of the bilateral R1 latencies in the blink reflex study and significant hypoesthesia in the two-point discrimination test compared to the control group (Table 2). These findings support the occurrence of subclinical cranial neuropathy caused by platinum-analogues chemotherapy.

In the present study, the chemotherapy group demonstrated a delay in the blink reflex including with bilateral $\mathrm{R} 1, \mathrm{R} 2 \mathrm{i}$, and R2c latencies compared to the control group, although statistical significance was found only in the bilateral R1 latencies (Table 2). In peripheral polyneuropathy caused by platinum agents, the dorsal root ganglia (DRG), which lacks an effective blood-brain barrier (BBB), are considered to be the primary targets of the drugs, which cause apoptosis of sensory neurons $[15,16]$. Similarly, in our patients, extra-axial lesions, including the sensory trigeminal roots, may be exposed to chemically-induced damage and, consequently, all the R1, R2i, and R2c latencies would probably be prolonged. In addition, Guney et al. [17] reported blink reflex alterations in diabetic patients, concluding that in the patients with extra-axial lesions, $\mathrm{R} 1$ is more sensitive than R2. Furthermore, the $\mathrm{R} 1$ response is known to be mainly conducted by exteroceptive, medium-thick myelinated A-beta $(A \beta)$ fibers, whereas the $\mathrm{R} 2$ response is predominantly conveyed by nociceptive, thin myelinated A-delta $(\mathrm{A} \delta)$ fibers [18]. In the previous studies of cisplatin neurotoxicity, large myelinated sensory nerve fibers were more vulnerable to chemotherapy and the decrement of mediumand large-sized neuron was observed in the patients' peripheral nerves [19-21]. We hypothesized that these results may be helpful in explaining the more profound change of R1 than R2 in our patients.

Because our patients did not show any symptoms or signs suggestive of a cranial neuropathy, we performed clinical evaluation with the two-point discrimination test as well as with electrodiagnostic studies. We found that the chemotherapy group showed significant hypoesthesia compared to the control group (Table 2). Thus, the cranial nerve involvement by platinum agents led to overt clinical manifestations, in addition to the elctrophysiological findings. In addition, given that mechanical stimulation including two-point discrimination sense was conducted through the $A \beta$ fibers, it could be interpreted as a consistent result with the more profound delay of R1 than R2 latencies in the chemotherapy group.

Neurotoxicity caused by platinum agents shows a cumulative dose effect. In the case of cisplatin, signs of neurotoxicity, such as a decrease or loss of DTR, begin to appear after a cumulative dose of greater than $300 \mathrm{mg} / \mathrm{m}^{2}$, and, therefore, most patients who finish chemotherapy with the usual dose $\left(300-450 \mathrm{mg} / \mathrm{m}^{2}\right)$ experience sensory neuropathy symptoms. In addition, after treatment with $750-850 \mathrm{mg} / \mathrm{m}^{2}$ of oxaliplatin, $82 \%-93 \%$ of patients experience neuropathic symptoms. It is known that the severity of neuropathy worsens as the cumulative dose of the drugs increase [22]. In subgroup analysis of the present study, the patients with polyneuropathy showed bilateral R1 and R2 delay compared to the control group, whereas those without the polyneuropathy showed only R1 delay. In addition, those with polyneuropathy showed significant delay of bilateral R1 and R2 latencies and had 
longer duration of chemotherapy and higher cumulative dose of platinum agents compared to those without the polyneuropathy (Table 4). It is likely that the platinum agents were neurotoxic to the patient's PNS, including the cranial nerves. Therefore, involvement of the cranial nerves may be more profound in patients with peripheral polyneuropathy and may be dependent on the cumulative dose of the drugs. On the other hand, the finding of the Rl delay of both the patients with and without the polyneuropathy may indicate that that $\mathrm{R} 1$ is more sensitive in detecting subclinical cranial neuropathy than is R2. In general, most peripheral polyneuropathies affect the lower limb nerves and sensory fibers before the upper limb nerves and motor fibers, respectively. However, that platinum-based chemotherapy may preferentially affect limb over cranial nerves has yet to be definitively defined. Similarly, Elkholy et al. [23] and Resende et al. [24] also reported blink reflex alterations not accompanied by peripheral polyneuropathy in patients with diabetes and end-stage renal disease.

The present study has some limitations. A major limitation was that the study design was cross-sectional and comparisons were made between patients and healthy controls. For future studies, comparison of the blink reflex before-and-after chemotherapy or, alternatively, between symptomatic and asymptomatic patients may be more ideal. However, most of the patients who underwent the chemotherapy were asymptomatic, even those with symptoms of peripheral polyneuropathy. Therefore, further study with before-and-after evaluations may reach a more valid conclusion. Secondly, the present study failed to obtain the homogeneity of chemotherapy regimen in the chemotherapy group. The most ideal study design would be to include patients who have received single agent chemotherapy participate in the chemotherapy group, but have most other patients on anti-cancer treatment receive combination chemotherapy, except for those who undergo chemotherapy with adjuvant treatment. In addition, patients who receive adjuvant chemotherapy receive lower cumulative doses of chemotherapeutic agents, and therefore, may not be appropriate as subjects of the study evaluating neurotoxicity. Accordingly, despite the use of combination chemotherapy, only patients who additionally used 5-FU or etoposide, which were known to have little neurotoxicity [22], were included as the subjects (in order to minimize the confounding effect of other chemothera- peutic agents). Thirdly, the chemotherapy group was divided into two subgroups according to whether they had peripheral polyneuropathy, and their cumulative dose of platinum agents was compared (Table 4). However, the usual dose of cisplatin (300-450 $\left.\mathrm{mg} / \mathrm{m}^{2}\right)$ and oxaliplatin $\left(\geq 700 \mathrm{mg} / \mathrm{m}^{2}\right)$ were greatly different from each other. For thease reasons, comparing their relative dose effect may not be appropriate. For the same reason, significant dose differences between the subgroups of cisplatin and oxaliplatin may not be adequately interpreted at face value (Table 3). Further research with larger numbers of patients comparing the dose for each chemotherapeutic agents (i.e., cisplatin or oxaliplatin) should be performed. Finally, the present study did not perform long-term follow-up observation of cranial neuropathy. Neuropathy caused by platinum agents may continue or worsen in the few weeks following completion of the chemotherapy, a phenomenon coined the coasting phenomenon [25]. Because we conducted electrodiagnostic studies and the two-point discrimination test within one month after finishing the chemotherapy, this strategy was effective for recruiting patients and preventing their loss to followup. Nonetheless, it remained difficult to judge whether evaluations were made after the cranial neuropathy sufficiently appeared. Accordingly, further research, including long-term follow-up observation by other known objective methods such as facial motor NCS, trigeminal somatosensory evoked potential, visual evoked potential, auditory evoked potential, vestibular evoked myogenic potential and masseter inhibitory reflex in company with blink reflex, is now indicated.

In the present study, comparison of the blink reflex study and two-point discrimination test demonstrated the likelihood of subclinical cranial neuropathy following platinum-analogues chemotherapy. Cranial neuropathy caused by the platinum agents was more profound in patients with peripheral polyneuropathy and may be dependent upon the cumulative dose of the drugs. The blink reflex presumably is of value in detecting subclinical cranial neuropathy in patients with platinum-analogues chemotherapy.

\section{CONFLICT OF INTEREST}

No potential conflict of interest relevant to this article was reported. 


\section{REFERENCES}

1. Kelland L. The resurgence of platinum-based cancer chemotherapy. Nat Rev Cancer 2007;7:573-84.

2. Wolf S, Barton D, Kottschade L, Grothey A, Loprinzi C. Chemotherapy-induced peripheral neuropathy: prevention and treatment strategies. Eur J Cancer 2008;44:1507-15.

3. Mollman JE. Cisplatin neurotoxicity. N Engl J Med 1990;322:126-7.

4. McKeage MJ. Comparative adverse effect profiles of platinum drugs. Drug Saf 1995;13:228-44.

5. Grothey A. Oxaliplatin-safety profile: neurotoxicity. Semin Oncol 2003;30(4 Suppl 15):5-13.

6. Verschraegen C, Conrad CA, Hong WK. Subacute encephalopathic toxicity of cisplatin. Lung Cancer 1995;13:305-9.

7. Sioka C, Kyritsis AP. Central and peripheral nervous system toxicity of common chemotherapeutic agents. Cancer Chemother Pharmacol 2009;63:761-7.

8. England JD, Gronseth GS, Franklin G, Miller RG, Asbury AK, Carter GT, et al. Distal symmetric polyneuropathy: a definition for clinical research: report of the American Academy of Neurology, the American Association of Electrodiagnostic Medicine, and the American Academy of Physical Medicine and Rehabilitation. Neurology 2005;64:199-207.

9. Higby DJ, Wallace HJ Jr, Albert DJ, Holland JF. Diaminodichloroplatinum: a phase I study showing responses in testicular and other tumors. Cancer 1974; 33:1219-5.

10. de Cos Escuin JS, Delgado IU, Rodriguez JC, Lopez MJ, Vicente CD, Miranda JA. Stage IIIA and IIIB non-small cell lung cancer: results of chemotherapy combined with radiation therapy and analysis of prognostic factors. Arch Bronconeumol 2007;43:358-65.

11. Goldberg RM, Sargent DJ, Morton RF, Fuchs CS, Ramanathan RK, Williamson SK, et al. A randomized controlled trial of fluorouracil plus leucovorin, irinotecan, and oxaliplatin combinations in patients with previously untreated metastatic colorectal cancer. J Clin Oncol 2004;22:23-30.

12. Amptoulach S, Tsavaris N. Neurotoxicity caused by the treatment with platinum analogues. Chemother Res Pract 2011;2011:843019.
13. Toker E, Yenice O, Ogut MS. Isolated abducens nerve palsy induced by vincristine therapy. J AAPOS 2004;8:69-71.

14. Vogel H, Horoupian DS. Filamentous degeneration of neurons: a possible feature of cytosine arabinoside neurotoxicity. Cancer 1993;71:1303-8.

15. Cavaletti G, Fabbrica D, Minoia C, Frattola L, Tredici G. Carboplatin toxic effects on the peripheral nervous system of the rat. Ann Oncol 1998;9:443-7.

16. McDonald ES, Windebank AJ. Cisplatin-induced apoptosis of DRG neurons involves bax redistribution and cytochrome c release but not fas receptor signaling. Neurobiol Dis 2002;9:220-33.

17. Guney F, Demir O, Gonen MS. Blink reflex alterations in diabetic patients with or without polyneuropathy. Int J Neurosci 2008;118:1287-98.

18. Esteban A. A neurophysiological approach to brainstem reflexes: blink reflex. Neurophysiol Clin 1999; 29:7-38.

19. Cavaletti G, Tredici G, Marmiroli P, Petruccioli MG, Barajon I, Fabbrica D. Morphometric study of the sensory neuron and peripheral nerve changes induced by chronic cisplatin (DDP) administration in rats. Acta Neuropathol 1992;84:364-71.

20. Hovestadt A, van der Burg ME, Verbiest HB, van Putten WL, Vecht CJ. The course of neuropathy after cessation of cisplatin treatment, combined with Org 2766 or placebo. J Neurol 1992;239:143-6.

21. Barajon I, Bersani M, Quartu M, Del Fiacco M, Cavaletti G, Holst JJ, et al. Neuropeptides and morphological changes in cisplatin-induced dorsal root ganglion neuronopathy. Exp Neurol 1996;138:93-104.

22. Miltenburg NC, Boogerd W. Chemotherapy-induced neuropathy: a comprehensive survey. Cancer Treat Rev 2014;40:872-82.

23. Elkholy SH, Hosny HM, Shalaby NM, El-Hadidy RA, Abd El-Rahim NT, Mohamed MM. Blink reflex in type 2 diabetes mellitus. J Clin Neurophysiol 2014;31:5525.

24. Resende LA, Caramori JC, Kimaid PA, Barretti P. Blink reflex in end-stage-renal disease patients undergoing hemodialysis. J Electromyogr Kinesiol 2002;12:159-63.

25. Quasthoff S, Hartung HP. Chemotherapy-induced peripheral neuropathy. J Neurol 2002;249:9-17. 